This is a author version of

D. Schlipf and P. W. Cheng,

"Flatness-based Feedforward Control of Wind Turbines Using Lidar", published in

Proceedings of the 19th World Congress of the International Federation of Automatic Control, Cape Town, South Africa, 2014.

\title{
Flatness-based Feedforward Control of Wind Turbines Using Lidar *
}

\author{
David Schlipf, Po Wen Cheng \\ Stuttgart Chair of Wind Energy, University of Stuttgart, Germany, \\ (david.schlipf/powen.cheng@ifb.uni-stuttgart.de)
}

\begin{abstract}
Current lidar technology is offering a promising opportunity to take a fresh look at wind turbine control. This work evaluates a flatness-based feedforward approach, that allows to calculate the control action based on trajectories of the rotor speed and tower motion using wind measurements. The trajectories are planned online considering actuator constrains to regulate the rotor speed and minimize tower movements. The feedforward signals of the collective pitch and generator torque update can be combined with conventional feedback controllers. This facilitates the application on commercial wind turbines. Simulations using a realistic lidar simulator and a full aero-elastic model show considerable reduction of tower and shaft loads.
\end{abstract}

Keywords: Feedforward control; trajectory planning; windmills; adaptive filters.

\section{INTRODUCTION}

The reduction of structural loads by advanced control is an important lever to lower the cost of wind energy. Traditional controllers with a rotational speed feedback react to changes in wind conditions with a delay due to the inertia of the rotor. LIDAR (LIght Detection And Ranging) technology allows the measurement of wind speeds in front of the wind turbine, which offers the possibility to integrate the preview information in new control concepts. Feedforward collective pitch control is able to reduce structural loads for high wind speeds and can be easily combined with traditional feedback controllers for real life applications [Schlipf et al., 2012, Scholbrock et al., 2013]. By solving an optimal control problem computationally more complex controllers can mitigate loads by adjusting blade pitch and generator torque also around rated wind speed, where most of the turbine's damage is accumulated [Körber and King, 2011, Schlipf et al., 2013b, Aho et al., 2013]. Flatness-based feedforward control is considered by Utz et al. [2007] to be a promising alternative for industrial applications.

The Tower EQUILibirum Accommodation (TEQUILA) introduced in this work combines the advantages of the approaches by making use of the flatness property of a reduced nonlinear wind turbine model. The control inputs are calculated with low computational effort based on the measured wind speed and are combined with conventional feedback control. The feedforward signal for collective pitch and generator torque allows load reduction in the transition region between partial and full power operation.

Section 2 and Section 3 summarize the modeling of the wind turbine and the lidar measurements. In Section 4 the flatness-based feedforward controller is derived and evaluated in Section 5. Section 6 concludes the paper.

\footnotetext{
* Part of this research is funded by the German Federal Ministry for the Environment, Nature Conservation and Nuclear Safety (BMU) in the framework of the German joint research project "LIDAR II".
}

\section{MODELING OF THE WIND TURBINE}

In this study a full and a reduced model of a $5 \mathrm{MW}$ reference turbine [Jonkman et al., 2009] are used.

\subsection{Full Simulation Model}

Simulations are done with the coupled aero-elastic simulation tool FAST (Fatigue, Aerodynamics, Structures, and Turbulence, [Jonkman and Buhl, 2005]). For onshore turbines the model consists of a flexible multibody system which experiences external forces from aerodynamics. The structural model represents dynamics of flexible parts such as tower, blades, and drive train up to the second mode. A second order linear model is added for the collective pitch actuator, resulting in a total of 16 degrees of freedom. In the aerodynamic subsystem two different disturbance inputs are implemented. Coherent time series of wind characteristics such as wind speed, direction, and shears are used for extreme load calculations. Fatigue simulations are done with a turbulent three-dimensional wind field over the rotor disk generated with TurbSim [Jonkman, 2009]. In both cases, aerodynamic forces along the blades are calculated iteratively by applying the Blade Element Momentum theory and transferred to the structural model. The described simulation tools have proven reliable accuracy which justifies its application as a full simulation model.

\subsection{Reduced Design Model}

For the flatness-based controller design the aero-elastic model cannot be used due to the complexity of the iterative calculation of the aerodynamics. Here, a reduced model is derived. It features several simplifications in a way that it still reproduces reliably the overall dynamic behavior of the system. The SLOW (Simplified Low Order Wind turbine) model has been originally designed for floating wind turbines (see [Sandner et al., 2012]). Similar to the full simulation model the onshore version consists of a reduced servo-elastic and aerodynamic module. 


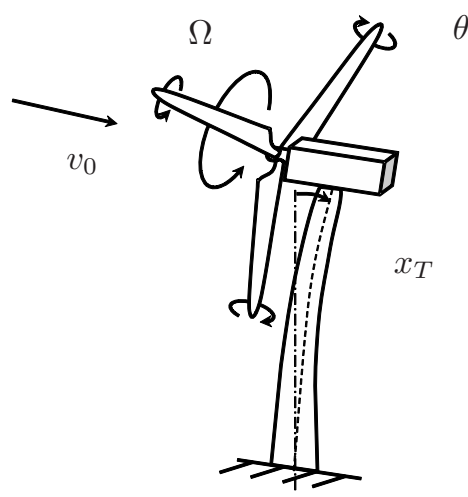

Fig. 1. Degrees of freedom for the reduced nonlinear model.

In the servo-elastic part only the first tower fore-aft bending mode and the rotational motion are considered:

$$
\begin{aligned}
J \dot{\Omega}+M_{g} / i & =M_{a} \\
m \ddot{x}_{T}+c \dot{x}_{T}+k x_{T} & =F_{a} \cos \left(\Theta_{N}\right) .
\end{aligned}
$$

Equation (1a) models the rotor dynamics, where $\Omega$ is the rotor speed, $M_{a}$ is the aerodynamic torque and $M_{g}$ the generator torque. Moreover, $i$ is the gearbox ratio and $J$ is the overall sum of the moments of inertia about the rotation axis. Equation (1b) describes the tower fore-aft dynamics, where $F_{a}$ is the aerodynamic thrust, $x_{T}$ the tower top fore-aft displacement, and $m, c$, and $k$ are the tower equivalent modal mass, structural damping, and bending stiffness, respectively. The nacelle pitching angle $\Theta_{N}$ is defined as sum of the shaft tilt angle $\Theta_{S}=5 \mathrm{deg}$ and the inclination of the nacelle due to the bending of the tower with length $h_{T}$ :

$$
\Theta_{N}=\Theta_{S}+\frac{3 x_{T}}{2 h_{T}}
$$

The pitch actuator and blades dynamics are modeled by a time delay $T_{B}$ from the demanded collective pitch angle $\theta_{c}$ to the actual collective pitch angle $\theta$ :

$$
\theta(t)=\theta_{c}\left(t-T_{B}\right) \text {. }
$$

In the aerodynamic part the aerodynamic torque and thrust acting on the rotor with the radius $R$ are

$$
\begin{aligned}
M_{a} & =\frac{1}{2} \rho \pi R^{3} \frac{c_{P}(\lambda, \theta)}{\lambda} v_{r e l}^{2} \\
F_{a} & =\frac{1}{2} \rho \pi R^{2} c_{T}(\lambda, \theta) v_{r e l}^{2},
\end{aligned}
$$

where $\rho$ is the air density, $\lambda$ the tip-speed ratio, defined as

$$
\lambda=\frac{\Omega R}{v_{\text {rel }}},
$$

and $c_{P}$ and $c_{T}$ are the effective power and thrust coefficients, respectively. Two dimensional look-up tables are used for the coefficients, which are obtained from steady state simulations with the full simulation model with a shaft tilt angle of $0 \mathrm{deg}$. The relative wind speed $v_{r e l}$ is used to model the aerodynamic damping and is defined as the superposition of the tower top speed and the rotor effective wind speed $v_{0}$ corrected with the nacelle pitching angle:

$$
v_{r e l}=\left(v_{0}-\dot{x}_{T}\right) \cos \left(\Theta_{N}\right)
$$

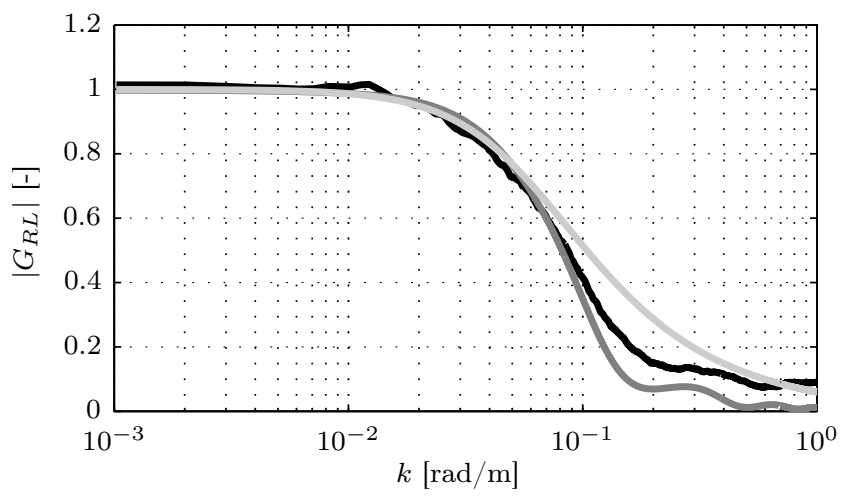

Fig. 2. Transfer functions between the rotor effective wind speed and lidar estimate: theoretical (dark gray) and from data (black). Fitted low pass filter (light gray).

\section{MODELING OF THE LIDAR MEASUREMENTS}

A preview of the rotor effective wind speed $v_{0}$ is necessary for the disturbance rejection with the TEQUILA controller. Current lidar technology provides the possibility to measure the speed of aerosols in front of the turbine by back-scattered light. Due to limitations in the lidar measurements and the wind evolution, only an estimate of the future rotor effective wind speed is possible [Schlipf et al., 2013a]. To consider most of these effects, the same generic wind field applied to the aero-elastic simulation in this work is scanned with a lidar simulator similar to [Schlipf et al., 2013b]. A pulsed system is simulated with a circular trajectory with six focus points in five focus distances linearly distributed between $R$ and $3 R$, which is currently tested in an experiment on a full scale wind turbine. The lidar simulation is evaluated at the nearest grid point of the generic wind field without interpolation to avoid overestimation of $v_{0}$. Taylor's frozen turbulence hypothesis, which assumes that the turbulent wind field moves unaffected with the average wind speed, is used in the simulation of the measurements as well as for the wind speed estimation. Here, all measurements are condensed to a lidar estimate of the rotor effective wind speed $v_{0 L}$ and $v_{0}$ is extracted from the turbulent wind field using the methods described by Schlipf et al. [2013b].

The transfer function $G_{R L}$ from $v_{0 L}$ to $v_{0}$ is calculated by

$$
G_{R L}=\frac{S_{R L}}{S_{L L}}
$$

where $S_{L L}$ is the auto spectrum of $v_{0 L}$ and $S_{R L}$ the cross spectrum between the two signals. Addition to $G_{R L}$ based on the simulated data, the theoretical function without wind evolution based on Schlipf et al. [2013a] is plotted in Figure 2. The agreement of both transfer functions demonstrates that the assumptions used within the lidar simulations are reasonable. A first order low pass filter is fitted to the transfer function, which maps $v_{0 L}$ to $v_{0}$ and filters out the uncorrelated frequencies to avoid wrong and unnecessary feedforward control action.

Contrary to Bossanyi [2012], wind evolution is not considered for convenience. However, the maximum coherent wavenumber (defined as cut-off frequency of the fitted filter) $\hat{k}=0.06 \mathrm{rad} / \mathrm{m}$ is realistic, see Schlipf et al. [2012]. 


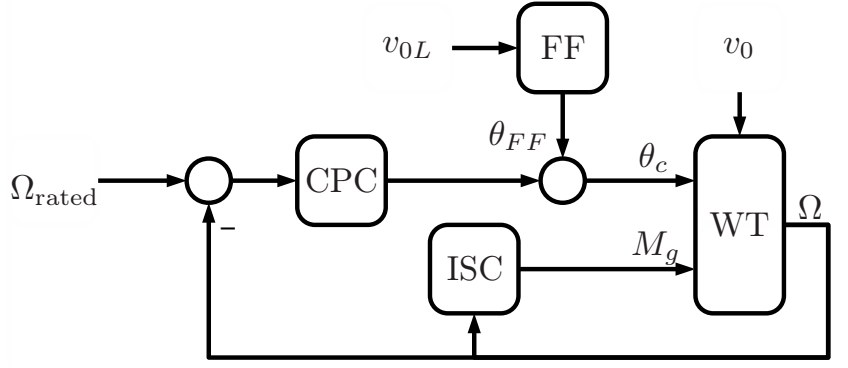

Fig. 3. Closed loop of the baseline feedforward controller.

\section{CONTROLLER DESIGN}

In this section a conventional baseline controller is described and the TEQUILA controller is derived.

\subsection{Baseline Controller}

The baseline controller (FB) is based only on feedback [Jonkman et al., 2009]. It combines a indirect speed controller (ISC) and a collective pitch controller (CPC), see Figure 3. Both controllers use the low pass filtered rotor speed $\Omega$ (or the generator speed $\Omega / i$ ) as input. The CPC regulates the rotor speed with the collective pitch angle demand $\theta_{c}$ to $\Omega_{\text {rated }}=12.1 \mathrm{rpm}$ using a gainscheduled PI controller. An anti-windup assures that the CPC only applies positive pitch angles. The ISC adjusts the generator torque $M_{g}$ with a nonlinear state feedback for an aerodynamically optimal operation as long as the blade pitch angle remains below a certain threshold $\theta_{f}=$ $1 \mathrm{deg}$, which is often referred to as "Region 2". The ISC regulates rated power above $\theta_{f}$, referred to as "Region 3 ".

\subsection{Tower Equilibrium Accommodation}

For the considered turbine and baseline controller the highest static aerodynamic thrust and thus the largest deflection is at the rated wind speed $v_{\text {rated }}=11.3 \mathrm{~m} / \mathrm{s}$, where the CPC starts to pitch the blades, see Figure 4. This results in high dynamic loading in the transition region, where the turbulent wind changes from below to above rated wind speed. The feedforward controller (FF) described in [Schlipf and Cheng, 2013] uses the derivative of the static pitch curve and the wind speed measurement $v_{0 L}$ when the turbulence hits the turbine. It compensate the effect of wind speed changes to the rotor speed in (1a), neglecting the effects of the ISC, see Figure 3. The benefit for the industrial application is, that only a simple update to common baseline controllers is necessary. At high wind speeds, holding $M_{a}$ constant results in smaller changes in $F_{a}$ and thus leads to tower load reduction. But the FF is only able to react, if the PI controller is not saturated. Furthermore, close to $v_{\text {rated }}$, holding $M_{a}$ constant results in high fluctuations of $F_{a}$ due to the nonlinearities in $c_{P}$ and $c_{T}$. Limiting the feedforward pitch rate close to $v_{\text {rated }}$ is a heuristic solution. Designing a feedforward controller holding $F_{a}$ directly constant would cause contradicting control goals to feedback and feedforward control.

A more direct solution is Nonlinear Model Predictive Control (NMPC), where a time series of the wind speed

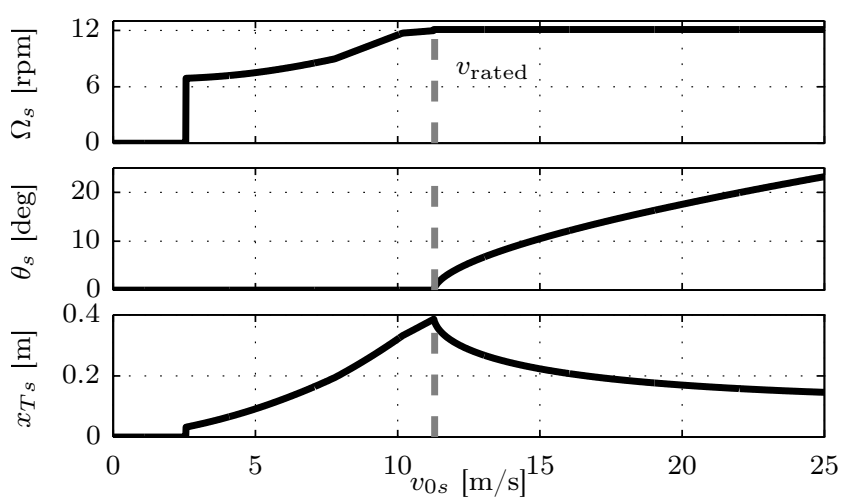

Fig. 4. Steady state values of the wind turbine model.

preview and a nonlinear model including the tower motion can be used to calculate repetitively an optimal trajectory for both, $\theta_{c}$ and $M_{g}$ and taking the actuator and state constraints into account. But solving the optimal control problem is computationally intensive and there is no guarantee to find the global minimum in the allotted time slot. Furthermore, the tower states $x_{T}$ and $\dot{x}_{T}$ have to be estimated, because only the acceleration signal $\ddot{x}_{T}$ is available on standard wind turbines. Aho et al. [2013] concentrates on the transition region and proposes the combination of offline calculation based on dynamic programming and an online Trajectory Tracking Controller (TTC). Using a model of the rotor motion, higher load reduction compared to the FF without the need of state estimation and the computational effort of the NMPC can be achieved. Both, NMPC and TTC may be the best solution in the future, but they have the drawback, that they replace the baseline feedback controller. This makes it less attractive to turbine manufacturers. Due to safety concerns industrial stateof-the-art is to extend the baseline controller by various additional control loops such as tower feedback damper and individual pitch control [Bossanyi et al., 2012].

The proposed TEQUILA combines the advantages of the FF (update on existing FB), the NMPC (considering tower deflection and wind preview over a time horizon, multivariable, considering constraints) and TTC (tracking of trajectories without extensive computational effort). It consists of a flatness-based feedforward controller and a trajectory planning algorithm and can be combined with a re-tuned feedback controller.

\subsection{The Flat Wind Turbine}

Flatness is a system property introduced by Fliess et al. [1995], that extends the concept of controllability from linear to nonlinear systems. A systems is flat, if a - not necessarily physical - so-called flat output exists such that all system states and inputs can be explicitly expressed in terms of the flat output and a finite number of its derivatives. This property can be used to plan input trajectory on a nonlinear system based on the flat output.

The wind turbine model (1)-(6) with the states $\Omega, x_{T}$, and $\theta$ and the control inputs $M_{g}$ and $\theta_{c}$ is a flat system with the flat outputs $x_{T}$ and $\Omega$. Thus, all states and the system inputs can be expressed by the desired trajectories $x_{T d}$ and $\Omega_{d}$, and their derivatives, which will be subsequently explained in detail. 


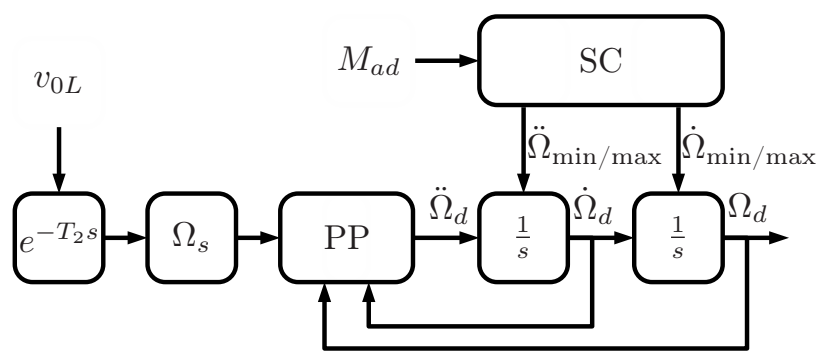

Fig. 5. Online trajectory planning for the rotor motion.

Using (2), (5), and (6), the desired tip speed ratio $\lambda_{d}$ is

$$
\begin{aligned}
\lambda_{d} & =\frac{\Omega_{d} R}{v_{\text {reld }}}, \\
\text { with } \Theta_{N d} & =\Theta_{S}+\frac{3 x_{T d}}{2 h_{T}} \\
v_{\text {reld }} & =\left(v_{0}-\dot{x}_{T d}\right) \cos \left(\Theta_{N d}\right) .
\end{aligned}
$$

Based on (1b) and (4b) the desired thrust coefficient is

$$
\begin{aligned}
c_{T d} & =\frac{2 F_{a d}}{\rho \pi R^{2} v_{r e l d}^{2}}, \\
\text { with } F_{a d} & =\frac{m \ddot{x}_{T d}+c \dot{x}_{T d}+k x_{T d}}{\cos \left(\Theta_{N d}\right)} .
\end{aligned}
$$

Using the inverse $\theta\left(\lambda, c_{T}\right)$ of the three-dimensional look-up table $c_{T}(\lambda, \theta)$, one obtains the third system state

$$
\theta_{d}=\theta\left(\lambda_{d}, c_{T d}\right) \text {. }
$$

Due to the time delay $T_{B}$ in (3), the future desired pitch angle is needed to calculate the desired demanded pitch angle $\theta_{c}$, which is the first system input:

$$
\theta_{c d}(t)=\theta_{d}\left(t+T_{B}\right) \text {. }
$$

Finally, the desired generator torque $M_{g d}$ - the second system input - can be obtained using (1a) and (4a).

$$
\begin{aligned}
M_{a d} & =\frac{1}{2} \rho \pi R^{3} \frac{c_{P}\left(\lambda_{d}, \theta_{d}\right)}{\lambda_{d}} v_{\text {reld }}^{2} \\
M_{g d} & =i\left(M_{a d}-J \dot{\Omega}_{d}\right) .
\end{aligned}
$$

This confirms, that the wind turbine model is flat with respect to the outputs $\Omega$ and $x_{T}$. There are unique trajectories for $M_{g d}$ and $\theta_{d}$ based on the desired trajectory for the tower and rotor states and the given wind speed $v_{0}$. For feedforward control the filtered and properly delayed lidar estimate $v_{0 L}$ is used. Continuously differentiable trajectories for $x_{T d}, \dot{x}_{T d}, \ddot{x}_{T d}, \Omega_{d}, \dot{\Omega}_{d}$ are designed in the next section to provide smooth control.

\subsection{Trajectory Planning}

Flatness based control is often used for trajectory planning to transfer a system from one equilibrium point to another. For wind turbine control an online trajectory planning of the flat output is necessary to account for the changes in wind conditions. In this work, the trajectory planning is based on a 2nd order system for the rotor motion and a 3rd order system for the tower motion following Zeitz [2012].

For the trajectory of the rotor motion (see Figure 5), the lidar measurement $v_{0 L}$ is first delayed by the buffer time

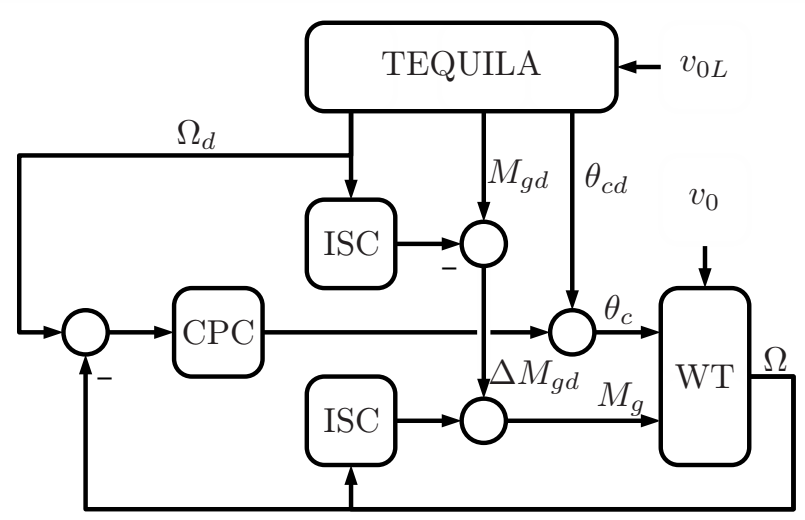

Fig. 6. Closed loop of the combined feedforward-feedback controller.

$T_{2}$ and then transferred to a rotor speed set point with the static function $\Omega_{S}$ (see Figure 4). The rotor motion is then shaped by the pole placement (PP). By choosing the two poles $p_{21}$ and $p_{22}$, the 2 nd derivative of the rotor speed is

$$
\ddot{\Omega}_{d}=p_{21} p_{22} \Omega_{s}+\left(p_{21}+p_{22}\right) \dot{\Omega}_{d}-p_{21} p_{22} \Omega_{d} \text {. }
$$

In the block system constraints (SC), the limits $\ddot{\Omega}_{\min / \max }$ and $\dot{\Omega}_{\min / \max }$ are calculated online based on $M_{a d}$ and its derivative from (12a) such that constraints for the generator torque $M_{g, \max } / \min$ and its rate $\dot{M}_{g, \max / \min }$ are not violated:

$$
\begin{aligned}
& \dot{\Omega}_{\min / \max }=\frac{M_{a d}}{J}-\frac{M_{g, \max / \min }}{i J} \\
& \ddot{\Omega}_{\min / \max }=\frac{\dot{M}_{a d}}{J}-\frac{\dot{M}_{g, \max / \min }}{i J}
\end{aligned}
$$

The desired rotor motion trajectories $\dot{\Omega}_{d}$ and $\Omega_{d}$ are then obtained by applying the constraints and successive integration following Graichen and Zeitz [2006]. The rotor dynamics are designed for low frequency tracking of the desired speed. The buffer time $T_{2}$ is set such that the rotor speed changes with the arrival of the wind field at the rotor.

The desired tower trajectories are planned in a similar way by choosing the buffer time $T_{3}$ and the poles $p_{31}, p_{32}$ and $p_{33}$ to minimize the tower motion and by considering the constraints of the pitch angle $\theta_{\min / \max }$ and rate $\dot{\theta}_{\min / \max }$.

\subsection{Combined Feedforward-Feedback Controller}

There are two main issues when combining the TEQUILA controller with the feedback controller as illustrated in Figure 6. First, all controllers need to have the same control objective. The common CPC has a constant reference value $\Omega_{\text {rated }}$, while the desired rotor speed $\Omega_{d}$ falls below this value for wind speeds below $v_{\text {rated }}$. This issue is solved by using the desired rotor speed $\Omega_{d}$ as reference signal.

The second issue is that the desired generator torque $M_{g d}$ cannot be simply applied to the nonlinear state feedback of the ISC. Therefore, $\Omega_{d}, \theta_{d}$, and the ISC are used to calculate a desired generator torque update $\Delta M_{g d}$. Furthermore, both outputs of the ISCs are filtered by a low pass filter to avoid large differences if both ISCs are not switching simultaneously between Region 2 and 3 . 

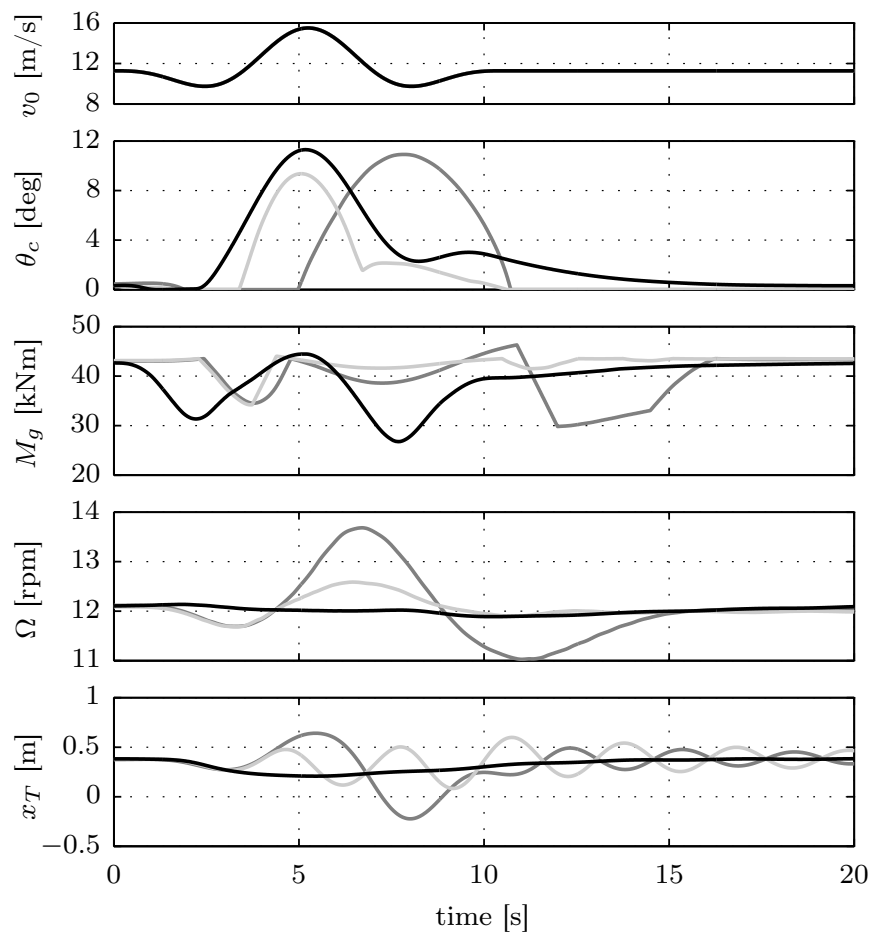

Fig. 7. Reaction to EOG at $v_{\text {rated }}$ FB (dark gray), $\mathrm{FB}+\mathrm{FF}$ (light gray), FB+TEQUILA (black).

\section{SIMULATION RESULTS}

In this section both combined feedforward-feedback controllers are compared to the feedback controller in aeroelastic simulations. First, perfect wind measurement is assumed and a coherent wind signal is used to estimate the benefit for extreme load reduction. Second, the wind information is based on the lidar simulator scanning a turbulent wind field for fatigue load investigations. The feedback controller is retuned separately to have the same rotor speed variation, if used along with one of the feedforward controllers.

\subsection{Extreme Loads}

In a first step the controllers are compared with regard to their reaction to an Extreme Operating Gust (EOG) at rated wind speed $v_{\text {rated. }}$. Figure 7 and Table 1 compare the demanded pitch angle $\theta_{c}$, generator torque $M_{g}$, tower top displacement $x_{T}$, rotor speed $\Omega$ for the different controllers. Both combined lidar assisted controllers are reacting prior to the $\mathrm{FB}$ alone, but only the TEQUILA minimizes significantly both rotor overspeed and maximum tower base fore-aft bending moment $M_{y T}$. Compared to the $\mathrm{FB}+\mathrm{FF}$ case, the flatness based controller provides both the pitch angle and the generator torque feedforward signal. As the desired rotor speed is used for the reference value, the controller is able to raise the pitch angle without the need to compensate a negative speed error.

Table 1. Maximum values for EOG simulation.

\begin{tabular}{ll||r|r|r} 
& & FB & FB + FF & FB+TEQUILA \\
\hline$\Omega$ & {$[\mathrm{rpm}]$} & 13.68 & 12.59 & 12.14 \\
$x_{T}$ & {$[\mathrm{~m}]$} & 0.64 & 0.60 & 0.38 \\
$M_{y T}$ & {$[\mathrm{MNm}]$} & 106 & 100 & 64
\end{tabular}
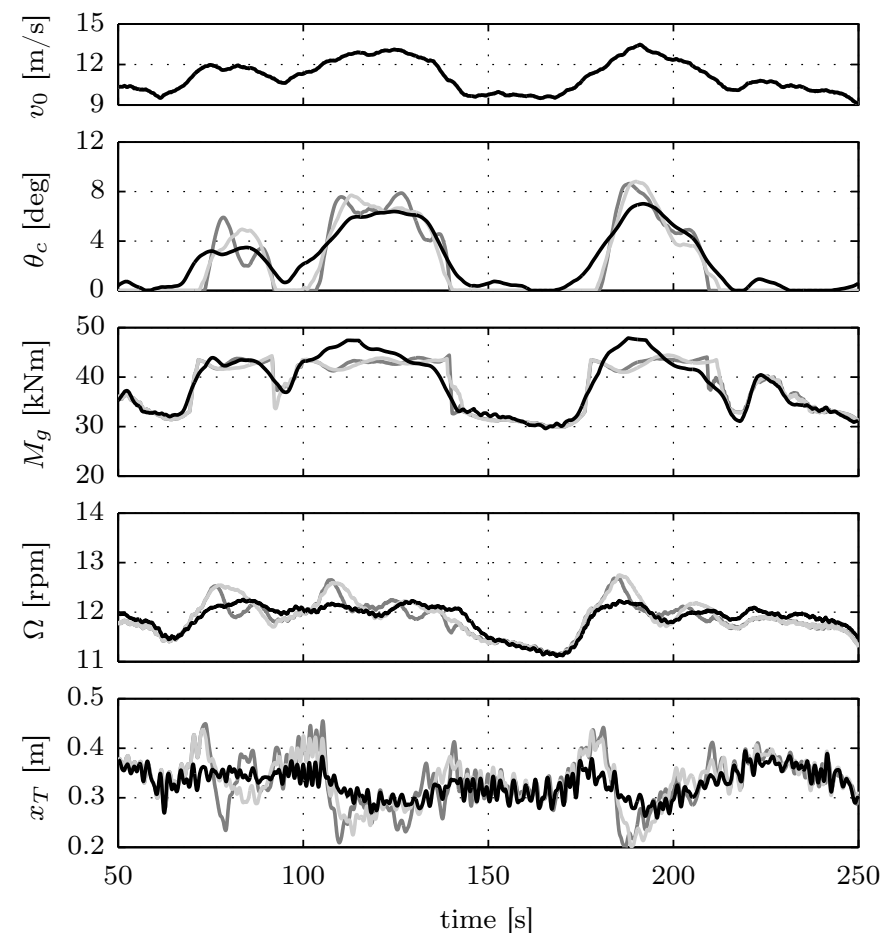

Fig. 8. Reaction to turbulent wind: FB (dark gray), $\mathrm{FB}+\mathrm{FF}$ (light gray), FB+TEQUILA (black).

Although the perfect measurement is unrealistic, the simulation shows, that TEQUILA using the internal SLOW model is robust with respect to model uncertainties when controlling the FAST model. Only small deviations from the desired trajectories are observed.

\subsection{Fatigue Loads}

In a second comparison the controllers are evaluated regarding their behavior in turbulent wind conditions. For this purpose a wind field with mean wind speed of $\bar{u}=12 \mathrm{~m} / \mathrm{s}$, a length of $3600 \mathrm{~s}$, and a turbulence intensity of $14.6 \%$ (IEC Class C) is used.

The exemplary section in Figure 8 depicts that TEQUILA is able to reduce significantly the oscillation of the tower compared to the FB only and the $\mathrm{FB}+\mathrm{FF}$ case - especially when the wind speed is crossing $v_{\text {rated }}$. Damage equivalent loads (DEL) of the tower base and of the low speed shaft bending moment $M_{L S S}$ can be alleviated with a reduced standard deviation of the pitch rate and without increasing the rotor speed variation or reducing the mean of the electrical power $P_{e l}$, see Table 2. Therefore, the flatness-based feedforward controller shows also promising performance using more realistic wind measurement and is able to outreach the baseline feedforward controller.

The decrease in loads is mainly due to compensation at low frequencies (see Figure 9). Oscillations at the tower eigenfrequency $(0.327 \mathrm{~Hz})$ and the blade passing frequency $(0.605 \mathrm{~Hz})$ are not affected. Due to the retuned feedback controller and the improved transition behavior with a rotor speed reference signal based on the wind information, the spectra can be reduced above the cut-off frequency $\hat{k} \bar{u} /(2 \pi)$ of the used low pass filter of the wind speed. 
Table 2. Results for fatigue loads simulation.

\begin{tabular}{ll||r|r|r} 
& & $\mathrm{FB}$ & $\mathrm{FB}+\mathrm{FF}$ & $\mathrm{FB}+\mathrm{TEQUILA}$ \\
\hline $\operatorname{DEL}\left(M_{y T}\right)$ & {$[\mathrm{MNm}]$} & 64.8 & 48.6 & 39.0 \\
$\operatorname{DEL}\left(M_{L S S}\right)$ & {$[\mathrm{MNm}]$} & 3.27 & 3.01 & 2.24 \\
$\operatorname{STD}(\dot{\theta})$ & {$[\mathrm{deg} / \mathrm{s}]$} & 0.622 & 0.325 & 0.199 \\
$\operatorname{STD}(\Omega)$ & {$[\mathrm{rpm}]$} & 0.289 & 0.281 & 0.285 \\
$\operatorname{MEAN}\left(P_{e l}\right)$ & {$[\mathrm{MW}]$} & 4.73 & 4.75 & 4.76
\end{tabular}
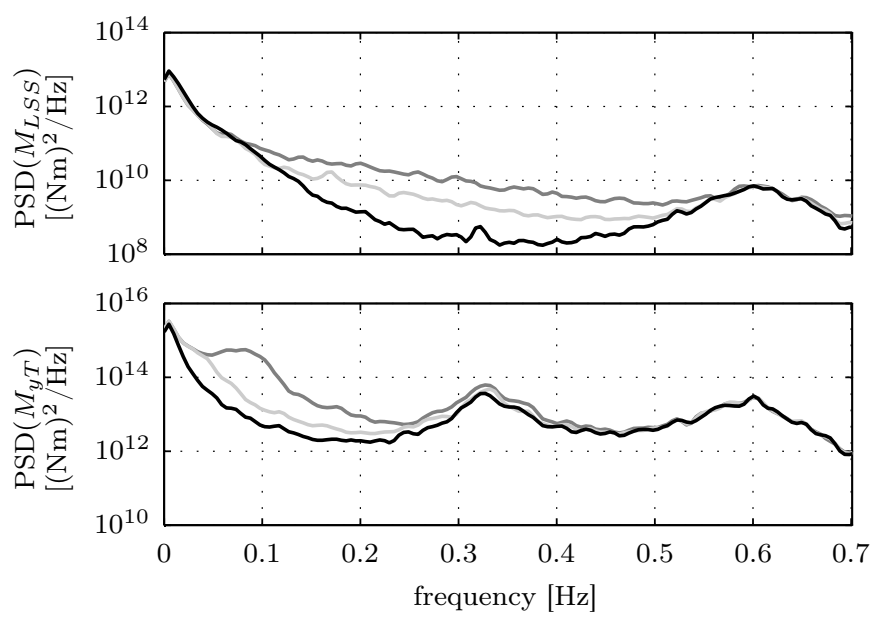

Fig. 9. Power Spectral Densities of FB (dark gray), $\mathrm{FB}+\mathrm{FF}$ (light gray), and FB+TEQUILA (black).

\section{CONCLUSION AND OUTLOOK}

This paper presents a flatness-based approach to improve wind turbine control based on the preview wind information provided by lidar technology. A reduced nonlinear model of a wind turbine is presented, which features the system property of differential flatness. Based on the wind speed of a simulated lidar device and considering the system constraints, trajectories of rotor speed and tower movement are continuously designed during operation and due to the flatness translated into trajectories for the control inputs pitch angle and generator torque. The trajectories are planned to hold the tower on its equilibrium manifold during the transition between partial and full load operations. The flatness-based feedforward controller is combined with a conventional feedback controller.

Simulations with a full aero-elastic model, turbulent wind, and a lidar simulator show promising performance in the presence of model and measurement uncertainties. Close to rated wind speed, load reduction on tower (30\%) and shaft $(40 \%)$ can be achieved.

In future work, a detailed simulation study over a full range of wind speeds is planned to investigate the robustness and to give a reliable estimation of the load reductions. This includes finding appropriate methods to enable and disable the flatness-based feedforward controller and to switch to the baseline feedforward controller for high wind speeds. If the promising results can be further confirmed, a field testing will be pursued.

\section{ACKNOWLEDGEMENTS}

Thanks to Ursula Smolka and Steffen Raach for the helpful discussions.

\section{REFERENCES}

J. Aho, L. Pao, and J. Hauser. Optimal trajectory tracking control for wind turbines during operating region transitions. In American Control Conference, Washington, USA, 2013.

E. Bossanyi. Un-freezing the turbulence: improved wind field modelling for investigating lidar-assisted wind turbine control. In European Wind Energy Association Annual event, Copenhagen, Denmark, 2012.

E. Bossanyi, B. Savini, M. Iribas, M. Hau, B. Fischer, D. Schlipf, T. van Engelen, M. Rossetti, and C. E. Carcangiu. Advanced controller research for multi-MW wind turbines in the UpWind project. Wind Energy, 15 (1):119-145, 2012.

M. Fliess, J. Lévine, P. Martin, and P. Rouchon. Flatness and defect of non-linear systems: introductory theory and examples. International Journal of Control, 61(6): 1327-1361, 1995.

K. Graichen and M. Zeitz. Inversion-based feedforward control design under input and output constraints. at Automatisierungstechnik, 54(4):187-199, 2006.

B. J. Jonkman. TurbSim user's guide. Technical Report NREL/TP-500-46198, September 2009.

J. Jonkman and M. L. Buhl. FAST user's guide. Technical Report NREL/EL-500-38230, NREL, August 2005.

J. Jonkman, S. Butterfield, W. Musial, and G. Scott. Definition of a 5-MW reference wind turbine for offshore system development. NREL/TP-500-38060, 2009.

A. Körber and R. King. Nonlinear model predictive control for wind turbines. In European Wind Energy Association Annual event, Brussels, Belgium, 2011.

F. Sandner, D. Schlipf, D. Matha, R. Seifried, and P. W. Cheng. Reduced nonlinear model of a spar-mounted floating wind turbine. In German Wind Energy Conference DEWEK, Bremen Germany, November 2012.

D. Schlipf and P. W. Cheng. Adaptive feed forward control for wind turbines. at-Automatisierungstechnik, 61(5): 329-338, 2013.

D. Schlipf, P. Fleming, F. Haizmann, A. Scholbrock, M. Hofsäß, A. Wright, and P. W. Cheng. Field testing of feedforward collective pitch control on the CART2 using a nacelle-based lidar scanner. In The Science of Making Torque from Wind, Oldenburg, Germany, 2012.

D. Schlipf, J. Mann, and P. W. Cheng. Model of the correlation between lidar systems and wind turbines for lidar assisted control. Journal of Atmospheric and Oceanic Technology, 30(10):2233-2240, 2013a.

D. Schlipf, D. J. Schlipf, and M. Kühn. Nonlinear model predictive control of wind turbines using LIDAR. Wind Energy, 16(7):1107-1129, 2013b.

A. Scholbrock, P. Fleming, L. Fingersh, A. Wright, D. Schlipf, F. Haizmann, and F. Belen. Field testing LIDAR based feed-forward controls on the NREL controls advanced research turbine. In Aerospace Sciences Meeting, Dallas, USA, 2013.

T. Utz, V. Hagenmeyer, and B. Mahn. Comparative evaluation of nonlinear model predictive and flatnessbased two-degree-of-freedom control design in view of industrial application. Journal of Process Control, 17 (2):129-141, 2007.

M. Zeitz. Feedforward control design in the frequency domain: Offline or online. at - Automatisierungstechnik, $60(7): 375-383,2012$. 